\title{
Recomendaciones de los Expertos para la Concientización del Asma en México
}

\section{Recommendations from Experts for Asthma Awareness in Mexico}

\author{
Francisco Cuevas-Schacht,* Javier Gómez-Vera, ${ }^{\ddagger}$ Erika del Carmen López-Estrada, \\ Gerardo Magdaleno-Maldonado," Juan Gabriel Maldonado-Hernández," Carol Vivian Moncayo-Coello, \\ María Isabel Rojo-Gutiérrez,**,*‡ Dolores Ochoa-Vázquez, ${ }^{\$ \S}$ Baltazar Pech-Alonso, IIII \\ Marlene Teresa Llópiz-Avilés," Antonieta Esperanza Boy-Ojeda," Andrés Alfredo Lezama-Armenta"
}

\footnotetext{
*Hospital Ángeles Lomas, Huixquilucan, Estado de Mexico, México;

${ }^{\ddagger}$ Hospital Regional Lic. Adolfo López Mateos, ISSSTE, Hospital Médica Sur, México;

$\S$ Instituto Nacional de Enfermedades Respiratorias Ismael Cosío Villegas, México;

${ }$ Hospital Central Militar, SEDENA, México; ${ }^{\top S}$ Secretaría de la Defensa Nacional, México;

**,*Hospital Juárez de México, México; ${ }^{\ddagger}$ Vicepresidente del Consejo Mexicano de Inmunología Clínica y Alergia;

${ }_{\$ \S}^{\S}$ Hospital General Gaudencio González Garza, Unidad Médica de Alta Especialidad La Raza, IMSS, México;

IIIICentro Médico Naval, México; Hospital Ángeles Acoxpa, México; " IIInstituto Médico de Capacitación, México.
}

RESUMEN. Introducción: El informe de estrategias de GINA (del inglés Global Initiative for Asthma) 2019 presenta los cambios más relevantes en el manejo del asma en los últimos 30 años. Objetivo: El propósito de este artículo es la difusión de los cambios en GINA 2019 con respecto al tratamiento del asma leve, así como expresar la postura del grupo de Expertos para la Concientización del Asma en México (ECAM), y sus recomendaciones a toda la comunidad médica tras el estudio minucioso de los cambios y la evidencia científica que los sustenta. Metodología: Se reunió a un grupo de médicos especialistas, líderes de opinión y expertos en asma en México con la finalidad de revisar, analizar y discutir los estudios y la evidencia que sustentan los cambios en GINA 2019. Resultados: Se definieron posturas y estrategias para abordar los diferentes retos que podrían presentarse debido a los cambios. Conclusiones: La recomendación es que todo paciente asmático debe tener tratamiento antiinflamatorio. Una estrategia en pacientes con síntomas intermitentes es utilizar una dosis baja de corticosteroide inhalado/formoterol por razón necesaria (PRN) como medicamento preferido de rescate; y en pacientes con síntomas persistentes, utilizar dosis bajas diarias de corticosteroides inhalados/ formoterol como tratamiento de control y de rescate PRN. Los resultados de los estudios y su impacto en la práctica clínica son prometedores.

Palabras clave: Asma, tratamiento antiinflamatorio, budesónida, formoterol, corticosteroides inhalados.

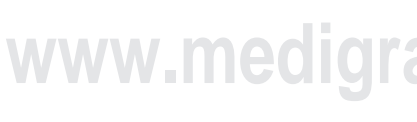

Correspondencia:

Dra. Marlene Teresa Llópiz-Avilés

Instituto Médico de Capacitación, México.

Correo electrónico: mllopiz@cromexicana.org;

mllopiz@hotmail.com

Trabajo recibido: 26-XI-2019; aceptado: 20-III-2020.
ABSTRACT. Introduction: The 2019 GINA strategy report presents the most relevant changes in asthma management in the last 30 years. Objective: The purpose of this article is to disseminate the changes in GINA 2019 regarding the treatment of mild asthma, and to express the position and recommendations from the members of the experts for awareness of asthma in Mexico. Methodology: A group of opinion leaders and asthma experts in Mexico was brought together to discuss the studies and evidence that support the changes in GINA 2019. Results: Positions and strategies were defined to address the different challenges posed by these changes. Conclusions: The recommendation is that every asthma patient should receive anti-inflammatory treatment. One strategy in patients with intermittent symptoms is to use a low-dose inhaled corticosteroid/formoterol as a preferred reliever, and daily low dose ICS/formoterol both as control and rescue (as needed) treatment for patients with persistent symptoms. The results of the studies and their impact on clinical practice are promising.

Keywords: Asthma, anti-inflammatory treatment, budesonide, formoterol, inhaled corticosteroids.

\footnotetext{
Abreviaturas:

$\mathrm{FEV}_{1} \quad=$ Forced expiratory volume in 1 second (volumen espiratorio forzado en el primer segundo).

GINA = Global Initiative for Asthma (iniciativa global para asma).

ICS = Inhaled corticosteroids (corticosteroides inhalados [CEI]).

LABA = Long acting beta agonist (beta agonista de acción prolongada).

OMS = Organización Mundial de la Salud.

PRN $=$ Por razón necesaria.

SABA = Short acting beta agonist (beta-agonista de acción corta).

BASALT $=$ Best adjustment strategy for asthma in long term

BEST = Beclomethasone and salbutamol treatment study.

TREXA $=$ Treating children to prevent exacerbations of asthma
} 


\section{INTRODUCCIÓN}

Según las estimaciones más recientes de la Organización Mundial de la Salud (OMS) y del informe de Global Initiative for Asthma (GINA), en el mundo, existen 300 millones de personas que padecen asma. Esto representa un problema de salud pública que afecta a todos los grupos etarios; tan sólo en 2017 hubo 383,000 muertes por asma en el mundo. ${ }^{1}$

El informe GINA se actualiza cada año, y cada cinco años se hace una revisión mayor, como esta última que está basada en evidencias internacionales. Los cambios propuestos en la actualización de 2019 son los más importantes para el manejo del asma en los últimos 30 años, pero irealmente es tan importante el cambio?, ¿qué cambió?, y ¿en qué benefician estos cambios a los pacientes con asma leve?

El grupo de Expertos para la Concientización del Asma en México (ECAM) determinó que estos cambios eran necesarios y - por fin- congruentes con la práctica clínica diaria. De acuerdo con GINA, se considera asma leve en todo aquél en el que se tenga un buen control con los pasos 1 o 2 de tratamiento. ${ }^{1}$

\section{Objetivo}

El propósito de este artículo es la difusión de los cambios en la actualización de 2019 del informe GINA con respecto al tratamiento del asma leve; asimismo, se busca expresar la postura y recomendaciones del ECAM a toda la comunidad médica tras el análisis minucioso de los cambios y la evidencia científica que los sustenta, además de proveer de herramientas a los médicos de primer contacto para dar el manejo inicial adecuado a los pacientes con asma leve que acuden a la consulta general y de urgencias.

\section{METODOLOGÍA}

Se reunió a un grupo de médicos expertos en asma que representan a diferentes instituciones de salud de México, con la finalidad de discutir los cambios en GINA 2019 con base en la revisión y análisis de la evidencia científica que sustenta dichas modificaciones.

A este grupo de trabajo se le nombró Expertos para la Concientización del Asma en México (ECAM). Para iniciar, se definió una serie de preguntas como punto de partida para discutir y extraer de esas respuestas los puntos clave, así como las conclusiones de esta reunión.

\section{¿Qué cambió en GINA 2019?}

Actualmente GINA 2019, por seguridad, no recomienda más la monoterapia con broncodilatadores de corta acción
(SABA), ya que existe evidencia de que, a pesar de dar alivio a corto plazo a la sintomatología del asma, su uso está asociado con el aumento del riesgo de exacerbaciones y el deterioro de la función pulmonar.

El tratamiento con SABA data de hace más de 50 años y se ha enfocado en resolver la broncoconstricción. Sin embargo, sabemos que la inflamación de la vía aérea es una condición constante en los pacientes asmáticos y que el no iniciar un tratamiento integral conlleva el riesgo de exacerbaciones y remodelación de las vías respiratorias. ${ }^{1}$

Es importante describir ambos mecanismos fisiopatológicos (el broncoespasmo y la inflamación) como parte de la enfermedad, porque ambos deben ser tratados desde que se establece el diagnóstico del paciente, independientemente de la gravedad con que se presenten.

GINA 2019 recomienda que todos los adultos y adolescentes con asma reciban un esquema de tratamiento con una dosis baja de corticosteroides inhalados (ICS)/formoterol por razón necesaria (PRN) en el paso 1 y con corticosteroides inhalados a dosis bajas diarias como tratamiento de mantenimiento o ICS/formoterol PRN en el paso 2.

Este manejo muestra mayor congruencia entre las guías y la práctica diaria, así como con la fisiopatología inflamatoria de la enfermedad, y permite que, desde el paso 1, el paciente se beneficie con la combinación de broncodilatador e ICS, abarcando así el control de la inflamación y de la broncoconstricción. Además, cuando la combinación incluye formoterol, existe la ventaja de un broncodilatador de inicio rápido.

El uso de dosis bajas de ICS reduce significativamente el número de hospitalizaciones y muertes relacionadas con asma y, de igual forma, es efectivo para la prevención de exacerbaciones graves, reducción de síntomas, mejoría de la función pulmonar y prevención del broncoespasmo inducido por ejercicio, incluso en aquellos pacientes con asma leve. ${ }^{2-4}$

Se recomienda iniciar, después de establecer el diagnóstico de asma, el tratamiento con un medicamento que contenga ICS tan pronto como sea posible. ${ }^{1}$

\section{¿Por qué el énfasis en los pacientes con asma leve?}

Hasta ahora, la investigación sobre asma se había enfocado en el manejo del paciente con asma grave, pero el enfoque actual es el de prevenir que los pacientes con asma leve lleguen a etapas avanzadas de la enfermedad. ${ }^{5,6}$

Existe evidencia que demuestra que los pacientes con asma leve tienen riesgo de exacerbaciones, incluso graves, y complicaciones relacionadas con la enfermedad. En el estudio realizado por Dusser, se menciona que, de la población con asma leve estudiada, de 30 a 37\% tienen riesgo de presentar una exacerbación aguda de asma, 16\% presentaron exacerbación asmática casi fatal y de 15 a 20\% murieron por asma. ${ }^{5}$ 
Esto está asociado con la dependencia de broncodilatadores de rescate y la falta de tratamiento de mantenimiento que controle el factor inflamatorio del asma.,

El tratamiento con ICS es el pilar del tratamiento del asma, ya que es una terapia local con baja tasa de efectos adversos y con buen perfil de seguridad y eficacia comprobada. Su uso está indicado como parte del tratamiento para asma, desde leve hasta grave.

\section{¿Qué provocó los cambios en GINA 2019?}

Desde 2007, GINA había buscado activamente intervenciones en asma leve en los pasos 1 y 2, con la finalidad de reducir el riesgo de exacerbaciones y muertes relacionadas con el asma, observadas con el uso de SABA como monoterapia. ${ }^{1}$

Múltiples estudios muestran que el uso de SABA como monoterapia implica mayores riesgos que beneficios en los pacientes asmáticos. Uno de ellos es el sobreuso de SABA, ya que provoca regulación a la baja del receptor beta adrenérgico, una disminución de la broncoprotección, hiperreactividad bronquial de rebote y una disminución en la respuesta al broncodilatador., ${ }^{7,8}$ Los cambios también obedecen a la creciente evidencia de los beneficios de la terapia combinada con ICS a dosis bajas y un broncodilatador en pacientes asmáticos con cualquier gravedad. ${ }^{2-4}$

Miembros investigadores de GINA buscaron realizar estudios aleatorizados controlados con el propósito de encontrar alternativas de tratamiento que beneficiaran al paciente. De aquí nace la idea para los estudios SYGMA 1, SYGMA 2 y Novel START (NS). ${ }^{2-4}$ De los resultados obtenidos en estos estudios, surgió la recomendación de utilizar dosis bajas de ICS/formoterol PRN o, como segunda opción, usar un ICS cada vez que se emplea un SABA PRN (si no se cuenta con la terapia combinada en un solo dispositivo), ya que se demostró que la administración de ICS a dosis baja reduce significativamente la tasa anual de exacerbaciones graves, el número de hospitalizaciones y las muertes relacionadas con asma. ${ }^{2-4}$

No es la primera vez que se intenta el manejo del paciente asmático con combinaciones de ICS y broncodilatadores; sin embargo, las dosis de ICS utilizadas anteriormente eran más elevadas y provocaban efectos secundarios propios de su clase, por lo que estos esquemas se abandonaron.

No obstante, los estudios BEST y TREXA demostraron una reducción en la tasa de exacerbaciones en comparación con el grupo que sólo recibía monoterapia y, en conjunto con el estudio BASALT, demostraron una tasa menor o igual de exacerbaciones en comparación con el grupo en tratamiento de mantenimiento con ICS. ${ }^{2-4}$

Novel START tiene un diseño más apegado a la realidad; en él, se encontró que la tasa de exacerbaciones en el grupo budesónida/formoterol fue más baja que en el grupo de albuterol, y no difirió de la tasa del grupo de budesónida. Esto demuestra que la terapia combinada produce mayor disminución en el riesgo de exacerbaciones graves en comparación con los otros. ${ }^{4}$

SYGMA I demostró la reducción de la exacerbación grave $<64 \%$ en el grupo de budesónida/formoterol PRN en comparación con el grupo de SABA PRN; la tasa del estudio Novel START fue $<60 \%$. En SYGMA I y II se demostró una diferencia no significativa en la reducción de exacerbaciones severas entre el grupo de budesónida/formoterol PRN y el grupo de budesónida (mantenimiento) más terbutalina PRN (rescate), en tanto que en Novel START se observaron menos exacerbaciones graves en el grupo budesónida/formoterol que en el grupo de budesónida (mantenimiento) más albuterol PRN (rescate). ${ }^{2-4}$ Cabe mencionar que, en México, la terbutalina no está disponible en el mercado.

Sin embargo, sería difícil evaluar el impacto de la terapia de ICS/formoterol como rescate en la función pulmonar, precisamente por el uso intermitente $y$, si el uso fuera prácticamente diario, entonces habría que replantear el cambio de escalón de tratamiento.

Los resultados de los estudios realizados con ICS a dosis baja/formoterol (evidencia a dosis baja únicamente con budesónida/formoterol) sustentan los cambios drásticos en la última actualización de GINA (2019). Al día de hoy, la recomendación es utilizar ICS/formoterol PRN como medicamento preferido de rescate, y si no se encuentra disponible, utilizar dosis bajas de ICS cuando se use SABA.

\section{Participación del paciente en el tratamiento}

Parte de la labor del médico es educar al paciente, pues para éste es difícil entender las razones del cambio en el tratamiento hacia uno que él no percibe tan efectivo como el que usaba antes. Se le deben explicar los riesgos y beneficios que tiene el nuevo esquema para que vea reflejado el beneficio en cuanto a su función pulmonar a largo plazo, así como el ahorro, el cual se traduce en un menor número de hospitalizaciones y la disminución significativa en el riesgo de muerte, consecuencias graves del sobreuso de SABA.

La falta de adherencia al tratamiento, en particular en el grupo con asma leve, tiene impacto en la evolución del paciente y el pronóstico a largo plazo. El paciente se «acostumbra» a su enfermedad y a utilizar la terapia de rescate ocasionalmente, de modo tal, que busca que no afecte sus actividades cotidianas, y cuando no tiene síntomas, suspende el tratamiento, lo cual sólo refleja que no comprende adecuadamente el carácter crónico de su enfermedad.

Se deben aclarar los términos y definiciones de manera puntual, especialmente en cuanto al concepto de control. En general, el paciente entiende por control el alivio de los síntomas con la terapia de rescate y limitación de 
actividades desencadenantes, pero se le debe explicar que control implica la ausencia de síntomas, una función pulmonar normal y un bajo riesgo de exacerbaciones.

Se debe reforzar la idea de que cuanto mayor sea su adherencia al tratamiento, mejores resultados tendrá. Parte de la educación del paciente consiste en hacer que comprenda que el asma es una enfermedad crónica y que ésta requiere un tratamiento permanente, aunque sus síntomas sean esporádicos.

Según la experiencia de ECAM, para el paciente no es relevante el número de viales de broncodilatador que utilice mientras no tenga síntomas y se «sienta bien». La frecuencia en la necesidad de terapia de rescate $y / o$ la cantidad de inhaladores que utilice el paciente son indicadores del nivel de inflamación en la vía aérea; sirven de referencia al profesional de la salud para evaluar la respuesta al tratamiento y el grado de control del asma, además de que pueden ser augurio de mal pronóstico a largo plazo.

El autocontrol en los pacientes con asma leve implica un plan de acción con educación en el uso de parámetros objetivos, como la flujometría, acompañada de un diario de eventos que permita al paciente saber cuándo se requiere observación y cuándo intervención. Sin este proceso de concientización sobre su enfermedad, resulta una decisión irresponsable dejar al paciente en libertad para decidir el uso de SABA PRN solo. Es primordial evitar ventanas de confusión entre el tratamiento de rescate y tratamiento de mantenimiento.

Dar a los pacientes el poder de identificar cuándo es buen momento para la administración del medicamento sería crucial para su evolución a largo plazo, ya que la resultante de un mal manejo es la pérdida de la función respiratoria o exacerbaciones de riesgo vital. Es importante que el paciente tenga un instructivo por escrito (Plan de Acción) para saber cómo proceder en caso de exacerbación.

\section{CONCLUSIONES}

El grupo de ECAM considera que las modificaciones en las guías GINA son necesarias y congruentes con la fisiopatología del asma, y justifican lo que ya se hacía en la práctica clínica de forma empírica. Podemos señalar que las principales recomendaciones derivadas de los cambios en GINA 2019 son:

- Todo paciente asmático debe tener tratamiento antiinflamatorio.

- De acuerdo con lo indicado en el paso 1, en pacientes con síntomas intermitentes, la estrategia es usar dosis bajas de ICS/formoterol PRN (evidencia actual únicamente con budesónida/formoterol) como tratamiento de rescate y de mantenimiento.
- En el paso 2, el controlador ideal es un ICS a dosis baja diaria de mantenimiento $y$, si no hay adherencia suficiente, una buena opción es ICS a dosis baja/ formoterol PRN.

Se debe trabajar para modificar la percepción de los pacientes con respecto a la monoterapia con SABA y promover las ventajas de la terapia combinada con ICS/ beta agonista de acción prolongada (LABA). La versatilidad de los fármacos combinados es que permiten utilizarlos como terapia de mantenimiento y terapia de rescate; y de igual forma, permiten moverse dentro de la guía en los diferentes grados de severidad de la exacerbación con un solo producto.

Los cambios en las recomendaciones de GINA tienen los objetivos de reducir las exacerbaciones graves y la mortalidad relacionada con asma leve, mejorar el control y reducir la progresión de la enfermedad. Los principales retos serán que el paciente con asma leve comprenda la cronicidad de su enfermedad, lograr la adherencia al tratamiento y modificar su dependencia de SABA como terapia de rescate, sustituyéndolo por ICS/LABA.

Aunque el grupo de ECAM tiene presente que todavía es muy temprano para hablar de un cambio en el paradigma del asma y que se tiene que dejar transcurrir el tiempo para observar evidencia más contundente sobre los beneficios de esta estrategia, los resultados preliminares, tanto en los estudios como en la práctica clínica, infieren buenos resultados.

\section{Agradecimientos}

Se agradece ampliamente al Instituto Médico de Capacitación (IMC) que se encargó de la organización y logística de ECAM, así como de la redacción y gestión del documento final.

\section{REFERENCIAS}

1. Global Initiative for Asthma. Pocket Guide for Asthma Management and Prevention (for adults and children older than 5 years) [accesado: 19 de julio de 2019]. Disponible en: https://ginasthma.org/wp-content/ uploads/2019/04/GINA-2019-main-Pocket-Guide-wms.pdf

2. O'Byrne PM, FitzGerald JM, Bateman ED, et al. Inhaled combined budesonide-formoterol as needed in mild asthma. (SYGMA 1). N Engl J Med 2018;378(20):1865-1876. https://doi.org/10.1056/ nejmoa1715274

3. Bateman ED, Reddel HK, O'Byrne PM, et al. As-needed budesonideformoterolversus maintenance budesonide in mild asthma. (SYGMA 2). N Engl J Med 2018;378(20):1877-1887. https://doi.org/10.1056/ nejmoa1715275

4. Beasley R, Holliday M, Reddel HK, et al. Controlled trial of budesonideformoterol as needed for mild asthma. (Novel Start). N Engl J Med 2019;380(21):2020-2030. https://doi.org/10.1056/nejmoa1901963

5. Dusser D, Montani D, Chanez P, et al. Mild asthma: an expert review on epidemiology, clinical characteristics and treatment 
recommendations. Allergy 2007;62(6):591-604. https://doi. org/10.1111/j.1398-9995.2007.01394.x

6. Reddel HK, Ampon RD, Sawyer SM, Peters MJ. Risks associated with managing asthma without a preventer: urgent healthcare, poor asthma control and over-the-counter reliever use in a crosssectional population survey. BMJ Open 2017;7(9):e016688. https:// doi.org/10.1136/bmjopen-2017-016688

7. Suissa S, Ernst P, Boivin JF, et al. A cohort analysis of excess mortality in asthma and the use of inhaled beta-agonists. Am J Respir Crit Care Med 1994;149(3 Pt 1):604-610. https://doi.org/10.1164/ajrccm.149.3.8118625

8. Hancox RJ, Cowan JO, Flannery EM, Herbison GP, McLachlan CR, Taylor DR. Bronchodilator tolerance and rebound bronchoconstriction during regular inhaled beta-agonist treatment. Respir Med 2000;94(8):767-771. https://doi.org/10.1053/rmed.2000.0820
Financiamiento: La reunión de expertos para la preparación de este documento y su publicación se realizaron a través del Instituto Médico de Capacitación, gracias al apoyo financiero irrestricto otorgado por AstraZeneca; y sin participación alguna en su desarrollo para asegurar que los especialistas pudieran expresar sus opiniones libremente y sin sesgos o conflicto de intereses. Los especialistas no recibieron ninguna compensación económica por su participación.

Conflicto de intereses: Los autores declaran no tener conflicto de intereses. 